\title{
The LUCIFER Project: Achievements and Near Future Prospects
}

\author{
J. W. Beeman' ${ }^{1}$. F. Bellini ${ }^{2,3}$ - P. Benetti ${ }^{4,5}$ - L. Cardani ${ }^{2,3}$ - N. Casali ${ }^{2,3}$.

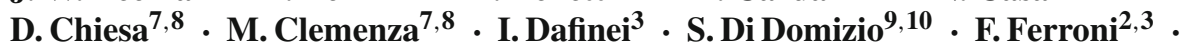 \\ L. Gironi ${ }^{7,8}$ - A. Giuliani ${ }^{11}$ - C. Gotti ${ }^{8}$ - M. Maino ${ }^{7,8}$ - S. S. Nagorny ${ }^{12}$.

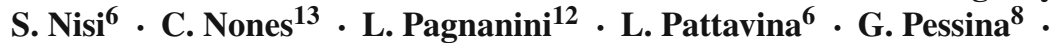 \\ G. Piperno $^{2,3}$-S. Pirro ${ }^{6}$ - E. Previtali ${ }^{8}$ - C. Rusconi $^{8}$ - K. Schäffner $^{6,12}$ (D) \\ C. Tomei $^{3}$ - M. Vignati $^{3}$
}

Received: 30 September 2015 / Accepted: 7 December 2015 / Published online: 6 January 2016 (C) Springer Science+Business Media New York 2016

\begin{abstract}
In the view of exploring the inverted hierarchy region future experiments investigating the neutrinoless double beta decay have to demand for detectors with excellent energy resolution and zero background in the energy region of interest. Cryogenic scintillating bolometers are very suitable detectors for this task since they provide particle discrimination: the simultaneous detection of the phonon and light signal allows us to identify the interacting type of particle and thus guarantees a suppression of $\alpha$-induced backgrounds, the key-issue for next-generation tonne-scale bolometric experiments. The LUCIFER project aims at running the first array of enriched scintillating $\mathrm{Zn}^{82} \mathrm{Se}$ bolometers (total mass of about $8 \mathrm{~kg}$ of ${ }^{82} \mathrm{Se}$ ) with a background level
\end{abstract}

K. Schäffner

karoline.schaeffner@lngs.infn.it

1 Lawrence Berkeley National Laboratory, Berkeley, CA 94720, USA

2 Dipartimento di Fisica, Sapienza Università di Roma, 00185 Roma, Italy

3 INFN - Sezione di Roma I, 00185 Roma, Italy

4 Dipartimento di Chimica, Università di Pavia, 27100 Pavia, Italy

5 INFN - Sezione di Pavia, 27100 Pavia, Italy

6 INFN - Laboratori Nazionali del Gran Sasso, 67010 Assergi (L'Aquila), Italy

7 Dipartimento di Fisica, Università di Milano-Bicocca, 20126 Milan, Italy

8 INFN - Sezione di Milano Bicocca, 20126 Milan, Italy

9 INFN - Sezione di Genova, 16146 Genova, Italy

10 Dipartimento di Fisica, Università di Genova, 16126 Genova, Italy

11 Centre de Spectrometrié de Masse, 91405 Orsay, France

12 Gran Sasso Science Institute, 67100 L'Aquila, Italy

13 CEA, Irfu, SPP Centre de Saclay, 91191 Gif-sur-Yvette, France 
as low as $10^{-3}$ counts/( $\mathrm{keV} \mathrm{kg} \mathrm{y)} \mathrm{in} \mathrm{the} \mathrm{energy} \mathrm{region} \mathrm{of} \mathrm{interest.} \mathrm{The} \mathrm{main} \mathrm{effort} \mathrm{is}$ currently focused on the finalization of the crystal growth procedure in order to achieve high quality $\mathrm{Zn}^{82} \mathrm{Se}$ crystals both in terms of radiopurity and bolometric properties. We present results from tests of such crystals operated at mK temperatures which demonstrate the excellent background rejection capabilities of this detection approach towards a background-free demonstrator experiment. Besides, the high purity of the enriched ${ }^{82}$ Se material allows us to establish the most stringent limits on the half-life of the double beta decay of ${ }^{82} \mathrm{Se}$ on excited levels.

Keywords Double beta decay - Cryogenic detector - Scintillating bolometer . Particle identification $\cdot$ Zinc selenide

\section{Introduction}

The neutrinoless double beta decay $(0 \nu \mathrm{DBD})[1]$ is an extremely rare process where the parent nucleus decays by the simultaneous emission of two beta-particles only. The decay implies Majorana nature [2] of the neutrino and thus requires the presence of physics beyond the Standard Model of particle physics. The evidence of $0 v$ DBD would prove that neutrinos are their own anti-particles that lepton number is not conserved and constraints would be set on the absolute mass scale of the neutrinos.

Low temperature bolometers fulfill the stringent requirement for the search of neutrinoless double beta decay: crystals with different DBD isotope can be grown, and multi-kg detectors [3] can be operated with excellent energy resolution of $0.1-0.2 \%$ at the $Q$-value of the decay [4]. While a large number of the isotope enhances the sensitivity of the experiment, a good energy resolution is indispensable to reduce the ultimate background from the $2 \nu \mathrm{DBD}$, the so far rarest experimentally observed nuclear decay with half lives in the range of $\left(10^{18}-10^{22}\right)$ years [5]. In case of discovery, the possibility to use the same detection approach but employing a different DBD isotope is a singular feature of the bolometric technique.

To date, bolometers in $0 v$ DBD searches were mainly using $\mathrm{TeO}_{2}$ crystals operated as single-channel detectors $[4,6,7]$. At present, radioactive surface contamination is identified as the main background component [8,9]: $\alpha$-particles can loose a fraction of their initial energy while passing through the bulk material before interacting in the bolometer, thereby possibly creating a flat background contribution within the energy region of interest for $0 \nu \mathrm{DBD}[10]$.

For next-generation bolometric tonne-scale 0vDBD experiments [11] a particle identification technique is of pivotal importance. An active rejection of background can be achieved by scintillating bolometers as used already in direct dark matter searches $[12,13]$. The detection of the thermal signal produced by a particle interaction in the bolometer and the simultaneously emitted scintillation light signal using a dedicated cryogenic light detector allows us to discriminate $\alpha$-particles from $\beta / \gamma$-events thanks to their different scintillation light yield (LY).

The LUCIFER collaboration (Low Underground Cryogenic Installation For Elusive Rates) aims at operating the first demonstrator of the scintillating bolometer approach within CUPID interest group (CUORE Upgrade with Particle IDentification, presently 
in course of formation $[14,15])$, employing enriched scintillating bolometers based on $\mathrm{Zn}^{82} \mathrm{Se}$ for the search of the $0 \nu \mathrm{DBD}$ of ${ }^{82} \mathrm{Se}$.

\section{The LUCIFER Project}

The comprehensive R\&D activity on scintillating bolometers for the search of the $0 \nu \mathrm{DBD}$ within the framework of LUCIFER was started about five years ago. Diverse inorganic crystal scintillators including $\mathrm{CdWO}_{4}, \mathrm{ZnMoO}_{4}$, and $\mathrm{ZnSe}$ were studied extensively while being operated as scintillating bolometers at $\mathrm{mK}$ temperatures in the CUORE/LUCIFER R\&D dilution refrigerator located at Laboratori Nazionali del Gran Sasso (LNGS), a deep underground site (3600 m w. e.) in central Italy [16].

For the next step, namely a small scale detector array to demonstrate the feasibility for setting up a background-free bolometric experiment, the LUCIFER collaboration selected $\mathrm{ZnSe}$ crystals enriched in ${ }^{82} \mathrm{Se} . \mathrm{Zn}^{82} \mathrm{Se}$ has three distinct advantages: the high $Q$-value of selenium (2997 keV [17]) suppresses most of the natural $\beta / \gamma$-background with the most intense $\gamma$-line at $2615 \mathrm{keV}$, and the comparatively high natural abundance $(8.73 \%$ [18]) together with the possibility to acquire the enriched isotope from the European company URENCO allowed us to have now at our disposal $15 \mathrm{~kg}$ of enriched ${ }^{82} \mathrm{Se}$ metal, respecting both the time schedule and funding of LUCIFER. ${ }^{1}$ Last but not least the high content of Se of $56 \%$ in the $\mathrm{ZnSe}$ matrix is of advantage since the experimental volume is a limited and valuable good in low temperature experimental set-ups.

\subsection{ZnSe Crystal}

Bolometric experiments are of modular structure. For the LUCIFER experiment, we aim at a total of $30 \mathrm{Zn}^{82} \mathrm{Se}$ crystals of cylindrical shape $(43.6 \mathrm{~mm}$ in diameter and $55 \mathrm{~mm}$ in height) and a weight of about $430 \mathrm{~g}$ each. In order to read the thermal signal NTDs (Neutron Transmutation Doped Thermistor) based on germanium are attached to each crystal by means of epoxy resin droplets. More information on the characteristics of NTDs and their readout can be found in [19].

Several natural ZnSe crystals from different crystal suppliers have been tested aforehand in order to carefully study bolometric properties, intrinsic radioactive contaminations and particle identification capabilities [20,21], the latter is discussed in the following section. With a natural $431 \mathrm{~g}$ crystal, we achieve an energy resolution of $(13.4 \pm 1.3) \mathrm{keV}$ (FWHM) at $2615 \mathrm{keV}$, the ${ }^{208} \mathrm{Tl}$-line.

The main background contribution expected in such scintillating bolometers are high energetic $\gamma$ s from ${ }^{214} \mathrm{Bi}(Q$-value at $3.2 \mathrm{MeV})$ and pile-up events from ${ }^{208} \mathrm{Tl}$ $\gamma$-cascades. From a long background run of $524 \mathrm{~h}$ we calculate the following contamination in such natural ZnSe crystal by examining the $\alpha$-spectrum: we find $(17.2 \pm 4.6) \mu \mathrm{Bq} / \mathrm{kg}$ of ${ }^{232} \mathrm{Th}$ and $(24.6 \pm 5.5) \mu \mathrm{Bq} / \mathrm{kg}$ of ${ }^{238} \mathrm{U}$, both in equilibrium with their daughters [19]. For a demonstrator experiment in an almost zero-background

\footnotetext{
1 Since gaseous $\mathrm{SeF}_{6}$ can be produced, the enrichment procedure of choice for selenium is centrifugal enrichment, a well established and the most cost-efficient enrichment technology.
} 
framework the tolerable limits for contributions from ${ }^{232} \mathrm{Th}$ and ${ }^{238} \mathrm{U}$ decay chains, in the crystal, are at few tens of $\mu \mathrm{Bq} / \mathrm{kg}$ only. Such low levels of intrinsic radioactive contaminations in the crystal demand for a very well understood and controlled way of crystal growth and high purity raw materials.

\subsection{Cryogenic Light Detector}

Each crystal is paired with a cryogenic light detector for means of scintillation light detection. A schema of a crystal and its light detector is shown in Fig. 1 left. LUCIFER light detectors consist of thin Ge wafers with $44.5 \mathrm{~mm}$ in diameter and $0.18 \mathrm{~mm}$ in thickness. One side of the disk is coated with $60 \mathrm{~nm}$ of $\mathrm{SiO}_{2}$ for better light absorption. As the crystal, also the light absorber is read out by a NTD, optimized in size for the light detector. The sensitivity of such macroscopic light absorbers is as good as $\sigma=60 \mathrm{eV}$ (baseline noise) .

The light yield (LY) depends on the nature of the interacting particle and is defined as the intrinsic energy detected in the light absorber in $\mathrm{keV}$ relative to the energy deposit in the crystal in $\mathrm{MeV}$. For $\beta / \gamma$-events in $\mathrm{ZnSe}$, we measure a $\mathrm{LY}_{\beta / \gamma}$ around $6.4 \mathrm{keV} / \mathrm{MeV}$. The LY for $\alpha$-particles is somewhat peculiar since it is measured to be around $26.6 \mathrm{keV} / \mathrm{MeV}$ [21]. Thus, $\alpha$-particles produce about a factor of 4 more in scintillation light than $\beta / \gamma$-events at same energy, a microscopically non understood feature and so far solely observed in ZnSe crystals. The inverse behavior of the scintillation yield for $\alpha$-particles bears the risk to feature leakage events from the $\alpha$-distribution into the $\beta / \gamma$-region and thus the region of interest for $0 \nu \mathrm{DBD}$. This can happen in case scintillation light from $\alpha$-events is lost for detection due to crystal imperfections, surface defects or not ideal light collection efficiency. However, such leakage $\alpha$-events can be rejected by pulse shape analysis of the light signal. A dedicated analysis algorithm [21] is able to infer differences in the pulse shape of the light signal of $\beta / \gamma$ - and $\alpha$-events providing, besides the light yield discrimination, a second option for a highly efficient way of particle identification and rejection.

\subsection{Enriched ${ }^{82} \mathrm{Se}$}

For the LUCIFER pilot experiment a total of $15 \mathrm{~kg}$ of ${ }^{82} \mathrm{Se}$ with an effective level of enrichment of $96.3 \%$ was produced at URENCO, Stable Isotope Group in Almelo, in the Netherlands. The enrichment process consists of three main steps: procurement of $\mathrm{SeF}_{6}$, centrifugal enrichment of ${ }^{82} \mathrm{SeF}_{6}$ and conversion of ${ }^{82} \mathrm{SeF}_{6}$ gas to ${ }^{82} \mathrm{Se}$ metal beads. A more detailed description can be found in [22].

In order to investigate the amount of chemical and radioactive contaminations in the enriched material, we performed material screenings using inductively coupled plasma mass spectrometry (ICP-MS) and $\gamma$-spectroscopy with HP-Ge, both carried out onsite at LNGS. Chemical impurities are of concern as they can harm the scintillation performance (mainly involving contributions from $\mathrm{V}, \mathrm{Cr}, \mathrm{Mn}, \mathrm{Fe}, \mathrm{Co}$, and $\mathrm{Ni}[22,23]$ ) and the bolometric performance of the bolometer by adding an additional contribution to its heat capacity (involving ferromagnetic and paramagnetic elements [24]). The 
measured concentrations of chemical impurities are at a level of hundreds of $10^{-9} \mathrm{~g} / \mathrm{g}$ and fulfill the requirements for the LUCIFER demonstrator [25].

The study of internal radioactive contaminations via $\gamma$-spectroscopy was carried out on a $2.5 \mathrm{~kg}$ sample of $96.3 \%$ enriched ${ }^{82} \mathrm{Se}$ metal beads measured for about 75 days in a dedicated HP-Ge detector [26]. As we find no evidence for contributions from the natural decay chains ${ }^{235 / 238} \mathrm{U}$ and ${ }^{232} \mathrm{Th}$ as well as from the common isotopes ${ }^{40} \mathrm{~K}$ and ${ }^{60} \mathrm{Co}$, we can set limits as low as few tens of $\mu \mathrm{Bq} / \mathrm{kg}$ for these nuclides. The only observed nuclide at a detectable level is ${ }^{75} \mathrm{Se}$ with $(110 \pm 40) \mu \mathrm{Bq} / \mathrm{kg}$ (measured in Oct. 2014). However, its short half live (119.8 days) and the low $Q$-value of $863.6 \mathrm{keV}$ render its contribution for the search of the $0 \nu \mathrm{DBD}$ of ${ }^{82} \mathrm{Se}$ in LUCIFER insignificant.

Having in hand a DBD emitter with the given purity of the LUCIFER ${ }^{82} \mathrm{Se}$ material opens the possibility to further scrutinize the $2 \nu \mathrm{DBD}$ of ${ }^{82} \mathrm{Se}$ on the excited levels $0_{1}^{+}$, $2_{1}^{+}$, and $2_{2}^{+}$of ${ }^{82} \mathrm{Kr}$. With our $2.5 \mathrm{~kg}$ sample of $96.3 \%$ enriched ${ }^{82} \mathrm{Se}$, we extend the sensitivity on the half-life of ${ }^{82} \mathrm{Se} 2 \nu \mathrm{DBD}$ of before mentioned transitions by about one order of magnitude in comparison to previous literature values [27,28]. The evaluated lower limits and detailed information on the applied analysis method can be found in [25].

\subsection{Zn ${ }^{82}$ Se Crystal Growth}

The enriched crystal production is a sequence of the following steps: production of synthesized $\mathrm{Zn}^{82} \mathrm{Se}$ powder, $\mathrm{Zn}^{82} \mathrm{Se}$ crystal growth and mechanical processing of the boule to meet its final dimensions.

The procurement of the high purity metal ${ }^{82} \mathrm{Se}$ and $\mathrm{Zn}$ is followed by a dedicated synthesis procedure applied to transform zinc and selenium to $\mathrm{Zn}^{82} \mathrm{Se}$ powder. In order to fit the final crystal quality criteria for the LUCIFER demonstrator stringent requirements on the synthesis process had to be defined.

The employed zinc is high purity zinc $(99.9995 \%, 15 \mathrm{~kg})$ acquired from the National Science Center KITP in Ukraine. With due care, we investigated the chemical and radioactive contaminations by the same means as applied for the ${ }^{82} \mathrm{Se}$ material [26]. We observe in the $12 \mathrm{~kg}$ sample no contributions from the natural decay chains ${ }^{235 / 238} \mathrm{U}$ and ${ }^{232} \mathrm{Th}$ within 34 days of measurement, but we detect the short-lived nuclide ${ }^{65} \mathrm{Zn}$ (244 days) at a level of $5 \mathrm{mBq} / \mathrm{kg}$ (Aug. 2014) due to cosmogenic activation. The synthesis and the crystal growth via standard Bridgman technique are carried out at ISMA Kharkov (Ukraine) and are planned to be finalized within December 2015. The machining and polishing of the $\mathrm{Zn}^{82}$ Se crystals will be carried out at LNGS instead.

\section{Perspective}

The production yield for the enriched crystals is foreseen to be better than $60 \%$. Thus LUCIFER will have available in its first small detector array, consisting of $30 \mathrm{Zn}^{82} \mathrm{Se}$ crystals $\left(96.3 \%\right.$ enrichment level), a total number of $\sim 5 \times 10^{25}{ }^{82}$ Se nuclei for the search of $0 \nu$ DBD. A schema of the detector array which will be hosted in the former CUORICINO [6] and CUORE-0 [4] cryostat at LNGS is shown in Fig. 1 right. The data taking of the LUCIFER detector array operated within the frame of CUPID is 


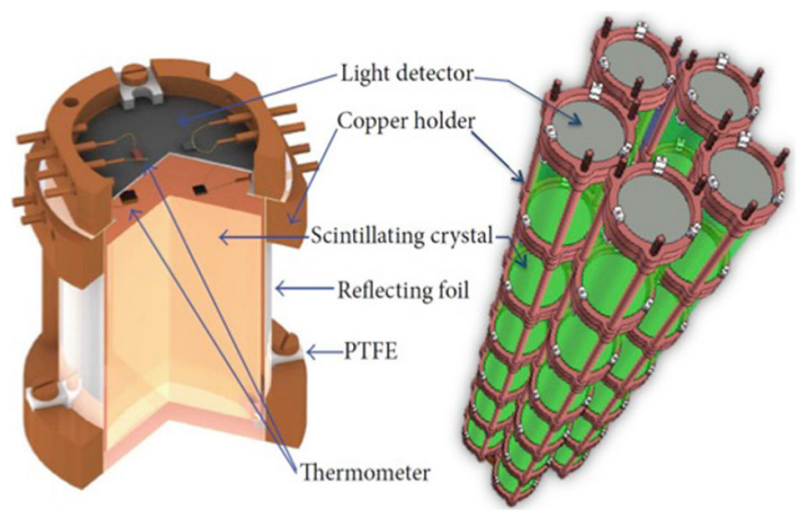

Fig. 1 Detailed schema of a ZnSe crystal and a cryogenic light detector is shown on the left. The array of 30 enriched $\mathrm{Zn}^{82} \mathrm{Se}$ crystals for the LUCIFER experiment is shown on the right (Color figure online)

planned to start end of 2016. To state a value for the expected half-life sensitivity of the LUCIFER demonstrator a Monte Carlo simulation was performed which allows us to evaluate the $0 v \mathrm{DBD}$ detection efficiency to be $76 \%$ and the expected background to be $\leq 1.5 \times 10^{-3}$ counts/( $\left.\mathrm{keV} \mathrm{kg} \mathrm{y}\right)$ in the energy region of interest. A live time of 2 years and detectors revealing energy resolutions as good as $10-20 \mathrm{keV}$ (FWHM) at the $Q$-value, will allow for an experiment in the almost zero-background framework, with only very few counts around the $Q$-value energy. Thus, the experimental goal of LUCIFER can be translated in a half-life sensitivity of $1.8 \times 10^{25}$ y (90 \% C.L.) considering 2 years of data taking.

\section{References}

1. F.T. Avignone, S.R. Elliott, J. Engel, Rev. Mod. Phys. 80, 481 (2008)

2. E. Majorana, Il Nuovo Cimento 14, 171 (1937)

3. L. Cardani et al., JINST 7, P01020 (2012)

4. D.R. Artusa et al., Eur. Phys. J. C 74, 2956 (2014)

5. A.S. Barabash, Nucl. Phys. A 935, 52 (2015)

6. E. Andreotti et al., Astropart. Phys. 34, 822-831 (2011)

7. C. Arnaboldi et al., Nucl. Instrum. Meth. A 518, 775-798 (2004)

8. F. Alessandria et al., Astropart. Phys. 45, 13-22 (2013)

9. M. Clemenza et al., Eur. Phys. J. C 71, 1805 (2011)

10. C. Bucci et al., Eur. Phys. J. C 2, 155-168 (2009)

11. D.R. Artusa et al., Eur. Phys. J. C 74, 3096 (2014)

12. G. Angloher et al., Eur. Phys. J. C 72, 1971 (2012)

13. G. Angloher et al., Eur. Phys. J. C 74, 3184 (2014)

14. CUPID interest group, arXiv:1504.03599 (2015)

15. CUPID interest group, arXiv:1504.0361 (2015)

16. M. Ambrosio et al., Phys. Rev. D 52, 3793 (1995)

17. D. Lincoln et al., Phys. Rev. Lett. 110, 012501 (2010)

18. M. Berglund, M.E. Wieser, Pure Appl. Chem. 83, 397-410 (2011)

19. J.W. Beemann et al., Adv. High Energy Phys. 2013, 237973 (2013). doi:10.1155/2013/237973

20. C. Arnaboldi et al., Astropart. Phys. 34, 344 (2011)

21. J.W. Beemann et al., J. Instrum. 8, P05021 (2013)

22. I. Dafinei, J. Cryst. Growth 393, 13-17 (2014) 
23. V. Ryzhikov et al., J. Cryst. Growth 364, 111-117 (2013)

24. T.O. Niinikoski et al., Europhys. Lett. 1, 499 (1986)

25. J.W. Beemann et al., arXiv:1508.01709 (2015), submitted to journal

26. G. Heusser et al., Radioactiv. Environm. 8, 495-510 (2006)

27. J. Suhonen et al., Z. Phys. A 358, 297-301 (1997)

28. R. Arnold et al., Nucl. Phys. A 636, 209-223 (1998) 